\title{
Linear Maps Which are Anti-derivable at Zero
}

\author{
Doha Adel Abulhamil ${ }^{1}$. Fatmah B. Jamjoom ${ }^{1}$. Antonio M. Peralta ${ }^{2}($ )
}

Received: 11 November 2019 / Revised: 16 February 2020 / Published online: 12 March 2020

(c) The Author(s) 2020

\section{Abstract}

Let $T: A \rightarrow X$ be a bounded linear operator, where $A$ is a $\mathrm{C}^{*}$-algebra, and $X$ denotes an essential Banach $A$-bimodule. We prove that the following statements are equivalent:

(a) $T$ is anti-derivable at zero (i.e., $a b=0$ in $A$ implies $T(b) a+b T(a)=0$ );

(b) There exist an anti-derivation $d: A \rightarrow X^{* *}$ and an element $\xi \in X^{* *}$ satisfying $\xi a=a \xi, \xi[a, b]=0, T(a b)=b T(a)+T(b) a-b \xi a$, and $T(a)=d(a)+\xi a$, for all $a, b \in A$.

We also prove a similar equivalence when $X$ is replaced with $A^{* *}$. This provides a complete characterization of those bounded linear maps from $A$ into $X$ or into $A^{* *}$ which are anti-derivable at zero. We also present a complete characterization of those continuous linear operators which are ${ }^{*}$-anti-derivable at zero.

Keywords $C^{*}$-algebra $\cdot$ Banach bimodule $\cdot$ Derivation $\cdot$ Anti-derivation $\cdot$ Maps anti-derivable at zero $\cdot$ Maps ${ }^{*}$-anti-derivable at zero

Mathematics Subject Classification Primary 46L05 - 46L57 · 47B47; Secondary $15 \mathrm{~A} 86$

Communicated by Mohammad Sal Moslehian.

$凶 \quad$ Antonio M. Peralta

aperalta@ugr.es

Doha Adel Abulhamil

dabulhamil0001@stu.kau.edu.sa

Fatmah B. Jamjoom

fjamjoom@kau.edu.sa

1 Present Address: Mathematics Departments, College of Sciences, King Abdulaziz University, P.O. Box 9039, Jeddah 21413, Saudi Arabia

2 Departamento de Análisis Matemático, Facultad de Ciencias, Universidad de Granada, 18071 Granada, Spain 


\section{Introduction}

Let us begin this note by formulating a typical problem in recent studies about preservers. Suppose $X$ is a Banach $A$-bimodule over a complex Banach algebra $A$. A derivation from $A$ to $X$ is a linear mapping $D: A \rightarrow X$ satisfying the following algebraic identity:

$$
D(a b)=D(a) b+a D(b), \quad \forall(a, b) \in A^{2} .
$$

A derivation $D$ is called inner if there exists $x_{0} \in X$ such that $D(a)=\delta_{x_{0}}(a)=\left[a, x_{0}\right]$ $=a x_{0}-x_{0}$ a for all $a \in A$.

A typical challenge on preservers can be posed in the following terms:

Problem 1 Suppose $T: A \rightarrow X$ is a linear map satisfying (1.1) only on a proper subset $\mathfrak{D} \subset A^{2}$. Is $T$ a derivation?

There is no need to comment that the role of the set $\mathfrak{D}$ is the real core of the question. A typical example is provided by the set $\mathfrak{D}_{z}:=\left\{(a, b) \in A^{2}: a b=z\right\}$, where $z$ is a fixed point in $A$. A linear map $T: A \rightarrow X$ is said to be a derivation at a point $z \in A$ if the identity (1.1) holds for every $(a, b) \in \mathfrak{D}_{z}$. In the literature, a linear map which is a derivation at a point $z$ is also called derivable at $z$.

Let us point out that there exist linear maps which are derivable at zero, but they are not derivations (for example, the identity mapping on a complex Banach algebra is a derivation at zero, but it is not a derivation).

If $T: A \rightarrow B$ is a linear mapping from $A$ into another Banach algebra satisfying $T(a b)=T(a) T(b)$ for all $(a, b) \in \mathfrak{D}_{z}$, we say that $T$ is a homomorphism at the point $z$. Linear maps which are Jordan $\left({ }^{*}\right.$-)derivations, or generalized $\left({ }^{*}\right.$-)derivations, or triple derivations, or (Jordan *)-homomorphisms at a point can be defined in similar terms. We understand that term "*-" is only employed when the involved structures are equipped with an involution.

Let us simply observe that a linear map $T$ between Banach algebras is a homomorphism at zero if and only if it preserves zero products (i.e., $a b=0$ implies $T(a) T(b)=0)$. We find in this way a natural link with the results on zero products preservers (see, for example, [1,2,8,10,28,29,32,33,47-51] for additional details and results). Burgos, Cabello-Sánchez and the third author of this note explore in [6] those linear maps between $C^{*}$-algebras which are *-homomorphisms at certain points of the domain, for example, at the unit element or at zero. We refer to [12,22,25,52-58] and [60] for additional related results.

According to the standard terminology (cf. $[1,4,7,9,23,30]$ ), we shall say that a linear operator $G$ from a Banach algebra $A$ into a Banach $A$-bimodule $X$ is a generalized derivation if there exists $\xi \in X^{* *}$ satisfying

$$
G(a b)=G(a) b+a G(b)-a \xi b(a, b \in A) .
$$

Every derivation is a generalized derivation; however, there exist generalized derivations which are not derivations. This notion is very useful when characterizing 
(generalized) derivations in terms of annihilation of certain products of orthogonal elements (see, for example, Theorem 2.11 in [4, §2]). The just quoted reference [4] contains an illustrative survey on local, 2-local and generalized derivations.

Let us revisit some recent achievements on maps derivable at certain points. For example, every continuous linear map $\delta$ on a von Neumann algebra is a generalized derivation whenever it is derivable at zero. If we additionally assume $\delta(1)=0$, we can conclude that $\delta$ is a derivation (see [25, Theorem 4]). Furthermore, for an infinitedimensional Hilbert space $H$, a linear map $\delta: B(H) \rightarrow B(H)$ which is a generalized Jordan derivation at zero, or at 1 , is a generalized derivation, even if $\delta$ is not assumed to be a priori continuous (cf. [24]). Zhu et al. prove in [59] a significant result showing that, for any Hilbert space $H$, a linear map $\delta: B(H) \rightarrow B(H)$ is a derivation if and only if it is a derivation at a nonzero point in $B(H)$ (see [34] for another related result).

Ghahramani and Pan [16] and Fadaee and Ghahramani [13] have recently considered certain variants of Problem 1 in their studies of continuous linear operators from a $C^{*}$-algebra $A$ into a Banach $A$-bimodule $X$ behaving like derivations or anti-derivations at elements in a certain subset of $A^{2}$ determined by orthogonality conditions. Let us detail the problem.

Problem 2 Let $T: A \rightarrow X$ be a continuous linear operator which is anti-derivable at zero, i.e.,

$$
T(a b)=T(b) a+b T(a) \text { for all }(a, b) \in \mathfrak{D}_{0} .
$$

Is $T$ an anti-derivation or expressible in terms of an anti-derivation?

Clearly, a mapping $\mathcal{D}: A \rightarrow X$ is called an anti-derivation if the identity (1.2) holds for every $(a, b) \in A^{2}$. If $A$ is a $C^{*}$-algebra, a *-derivation (respectively, a *-anti-derivation) from $A$ into itself, or into $A^{* *}$, is a derivation (respectively, an antiderivation) $d: A \rightarrow A$ satisfying $d\left(a^{*}\right)=d(a)^{*}$ for all $a \in A$.

Concerning Problem 1, Fadaee and Ghahramani prove in [13, Theorem 3.1] that for a continuous linear map $T: A \rightarrow A^{* *}$, where $A$ is a $C^{*}$-algebra, the following statements hold:

(a) $T$ is derivable at zero if and only if there is a continuous derivation $d: A \rightarrow A^{* *}$ and an element $\eta \in \mathcal{Z}\left(A^{* *}\right)$ (the center of $A^{* *}$ ) such that $T(a)=d(a)+\eta a$ for all $a \in A$;

(b) $T$ is $\mathrm{r}^{*}$-derivable at zero (that is, $a b^{*}=0 \Rightarrow a T(b)^{*}+T(a) b^{*}=0$ ) if and only if there is a continuous *-derivation $d: A \rightarrow A^{* *}$ and an element $\eta \in A^{* *}$ such that $T(a)=d(a)+\eta a$ for all $a \in A$ ( $\eta$ need not be central).

Ghahramani and Pan also considered a variant of Problem 1 in [16] in the context of (complex Banach) algebras which are zero product determined. We recall that an algebra $A$ is called zero product determined if for every linear space $Y$ and every bilinear map $V: A \times A \rightarrow Y$ satisfying $V(x, y)=0$ for every $x, y \in A$ with $x y=0$, there exists a linear map $T: A \rightarrow Y$ such that $V(x, y)=T(x y)$ for all $x, y \in A$. Brešar showed in [5, Theorem 4.1] that every unital algebra $A$ (algebraically) generated by its idempotents is zero product determined. Since this is the case of $B(H)$ for any infinite-dimensional complex Hilbert space $H$ (see [39, Theorem 1]), a property which is also enjoyed by properly infinite von Neumann algebras [39, Theorem 4], BunceDeddens algebras, irrotational rotation algebras, simple unital $\mathrm{AF}^{*}$-algebras with 
finitely many extremal states, UHF C*-algebras, unital simple $\mathrm{C}^{*}$-algebras of real rank zero with no tracial states [35, Corollary 4.9], [38, Theorem 4.6], properly infinite $C^{*}$ algebras [31, Corollary 2.2], and von Neumann algebras of type $\mathrm{II}_{1}$ [18, Theorem 2.2(a)], all these algebras are zero product determined.

A Jordan algebra is a non-necessarily associative algebra $B$ over a field whose multiplication, denoted by o, is commutative and satisfies the so-called Jordan identity:

$$
(a \circ b) \circ a^{2}=a \circ\left(b \circ a^{2}\right)(a, b \in B) .
$$

Every associative algebra is a Jordan algebra when equipped with the natural Jordan product given by $a \circ b=\frac{1}{2}(a b+b a)$. A Jordan derivation from $B$ into a Jordan $B$ module $X$ is a linear mapping $D: B \rightarrow X$ satisfying $D(a \circ b)=D(a) \circ b+a \circ D(b)$ for all $a, b \in B$. For the basic background on Jordan algebras, Jordan modules and Jordan derivations, the reader is referred to [20,21] and the references therein.

In what concerns Problem 1, Ghahramani and Pan proved in [16, Theorem 3.1] that for any zero product determined unital *-algebra $A$, and every linear mapping $T: A \rightarrow A$, the following statements hold:

(i) $T$ is derivable at zero if and only if there is a derivation $d: A \rightarrow A$ and an element $\eta \in \mathcal{Z}(A)$ such that $T(a)=d(a)+\eta a$ for all $a \in A$;

(ii) $T$ is $\mathrm{r}^{*}$-derivable at zero (that is, $a b^{*}=0 \Rightarrow a T(b)^{*}+T(a) b^{*}=0$ ) if and only if there is a ${ }^{*}$-derivation $d: A \rightarrow A$ and an element $\eta \in A$ such that $T(a)=d(a)+\eta a$ for all $a \in A$ ( $\eta$ need not be central).

When considering Problem 2 and maps which are anti-derivable at zero, the available conclusions are less determinate. Concretely, assuming that $A$ is a $\mathrm{C}^{*}$-algebra, Theorem 3.3 in [13] proves that for any continuous linear map $T: A \rightarrow A^{* *}$, the following statements hold:

(i) If $T$ is anti-derivable at zero, there is a continuous derivation $d: A \rightarrow A^{* *}$ and an element $\eta \in \mathcal{Z}\left(A^{* *}\right)$ such that $T(a)=d(a)+\eta a$ for all $a \in A$;

(ii) If $T$ is $\mathrm{r}^{*}$-anti-derivable at zero (i.e., $a b^{*}=0$ in $A \Rightarrow T(b)^{*} a+b^{*} T(a)=0$ ), there is a continuous *-derivation $d: A \rightarrow A^{* *}$ and an element $\eta \in A^{* *}$ such that $T(a)=d(a)+a \eta$ for all $a \in A(\eta$ need not be central).

If $A$ is a zero product determined unital ${ }^{*}$-algebra and $T: A \rightarrow A$ is a linear mapping, Theorem 3.4 in [16] proves the following statements:

(i) If $T$ is anti-derivable at zero, there is a Jordan derivation $d: A \rightarrow A$ and an element $\eta \in \mathcal{Z}(A)$ such that $T(a)=d(a)+\eta a$ for all $a \in A$;

(ii) If $T$ is $\mathrm{r}^{-}$-anti-derivable at zero, there is a Jordan ${ }^{*}$-derivation $d: A \rightarrow A$ and an element $\eta \in A$ such that $T(a)=d(a)+a \eta$ for all $a \in A$ ( $\eta$ need not be central).

In view of the previous result, it is natural to ask whether there exists a full characterization of those (continuous) linear maps which are $\left({ }^{*}\right.$-)anti-derivable at zero in pure algebraic terms. The main aim of this note is to complete our knowledge on these classes of continuous linear maps and to fill a natural gap which has not been fully covered. Our first main conclusion is contained in Theorem 6 where it is established that for each bounded linear operator $T$ from a $C^{*}$-algebra $A$ into an essential Banach $A$-bimodule $X$, the following statements are equivalent: 
(a) $T$ is anti-derivable at zero;

(b) There exist an anti-derivation $d: A \rightarrow X^{* *}$ and an element $\xi \in X^{* *}$ satisfying $\xi a=a \xi, \xi[a, b]=0, T(a b)=b T(a)+T(b) a-b \xi a$, and $T(a)=d(a)+\xi a$, for all $a, b \in A$.

It is further shown that if $A$ is unital, or if $X$ is a dual Banach $A$-bimodule, statement (b) above can be replaced with

$\left(\mathrm{b}^{\prime}\right)$ There exist an anti-derivation $d: A \rightarrow X$ and an element $\xi \in X$ satisfying $\xi a=a \xi, \xi[a, b]=0, T(a b)=b T(a)+T(b) a-b \xi a$, and $T(a)=d(a)+\xi a$, for all $a, b \in A$.

A similar conclusion holds when $X$ is replaced with $A^{* *}$.

In Sect. 4, we consider a $C^{*}$-algebra $A$ and an essential Banach $A$-bimodule equipped with an $A$-bimodule involution $*$ (i.e., a continuous conjugate linear mapping $x \mapsto x^{*}$ satisfying $\left(x^{*}\right)^{*}=x,(a x)^{*}=x^{*} a^{*}$ and $(x a)^{*}=a^{*} x^{*}$, for all $a \in A, x \in X)$. We give several natural examples of bimodule involutions. A Banach $A$-bimodule equipped with an $A$-bimodule involution will be called a Banach ${ }^{*}-A$ bimodule. Suppose $T: A \rightarrow X$ is a linear mapping from a $C^{*}$-algebra into a Banach *-A-bimodule. We shall say that $T$ is $\mathrm{r}^{*}$-anti-derivable at zero (respectively, - $^{*}$-antiderivable at zero) if $a b^{*}=0$ in $A$ implies $T(b)^{*} a+b^{*} T(a)=0$ in $X$ (respectively, $a^{*} b=0$ in $A \Rightarrow T(b) a^{*}+b T(a)^{*}=0$ in $\left.X\right)$.

Let $T: A \rightarrow X$ be a bounded linear operator where $A$ is a $C^{*}$-algebra and $X$ is an essential Banach ${ }^{*}$ - $A$-bimodule. In Theorem 9 , we prove the equivalence of the following statements:

(a) $T$ is $\mathrm{r}^{*}$-anti-derivable at zero (i.e., $a b^{*}=0$ in $A \Rightarrow T(b)^{*} a+b^{*} T(a)=0$ in $X$ ); (b) There exists a ${ }^{*}$-derivation $d: A \rightarrow X^{* *}$ and an element $\xi \in X^{* *}$ satisfying the following properties:

(i) $d([a, b])+[a, b] \xi+\xi^{*}[a, b]=0$, for all $a, b \in A$;

(ii) $T(a b)=a T(b)+T(a) b-a \xi b$, and $T(a)=d(a)+a \xi$ for all $a, b \in A$.

The conclusion in $(b)$ can be improved if $A$ is unital or if $X$ is a dual Banach $A$-bimodule. Finally, a complete characterization of those bounded linear operators $T: A \rightarrow X$ which are 1-*-anti-derivable at zero is presented in Theorem 11 (see also Corollary 12).

In Sect. 2, we take a closer look at anti-derivations from a general $C^{*}$-algebra $A$ into a Banach $A$-bimodule. Theorem 4 shows that these maps are in general very scarce.

\section{Are there Anti-Derivations on C*-Algebras?}

If one is interested on the study of linear maps from a $C^{*}$-algebra $A$ into a Banach $A$-bimodule which are anti-derivable at zero, a first natural step is to explore the class of anti-derivations on $\mathrm{C}^{*}$-algebra. For this purpose, we initiate our study by paying some attention to anti-derivations. An anti-derivation from an (associative) algebra $A$ into a Banach $A$ bimodule $X$ is a linear mapping $d: A \rightarrow X$ satisfying $d(a b)=d(b) a+b d(a)$ for all $(a, b) \in A^{2}$. An example seems to be welcome. Let 
us fix an element $x_{0} \in X$ satisfying

$$
x_{0}[a, b]=[a, b] x_{0} .
$$

for all $a, b \in A$, where $[a, b]=(a b-b a)$ denotes the Lie product or commutator of $a$ and $b$. The prototype of derivation from $A$ into $X$ is given by $\delta_{x_{0}}: A \rightarrow X, \delta_{x_{0}}(a)=$ $\left[a, x_{0}\right](a \in A)$. The assumption (2.1) implies that $a b x_{0}-x_{0} a b=b a x_{0}-x_{0} b a$ and thus

$$
\begin{aligned}
\delta_{x_{0}}(a b) & =a b x_{0}-x_{0} a b=b a x_{0}-x_{0} b a=b x_{0} a-x_{0} b a+b a x_{0}-b x_{0} a \\
& =\delta_{x_{0}}(b) a+b \delta_{x_{0}}(a)
\end{aligned}
$$

for all $a, b \in A$, witnessing that $\delta_{x_{0}}$ is a derivation and an anti-derivation. But, does such an element $x_{0}$ exist with the additional property that $\delta_{x_{0}} \neq 0$ ? Let us observe that $\delta_{x_{0}}$ also satisfies the following property:

$$
\delta_{x_{0}}([a, b])=\left[[a, b], x_{0}\right]=0 \text {, for all } a, b \in A .
$$

We shall see next that the identity in (2.2) actually characterizes anti-derivations.

Lemma 3 Let $\delta: A \rightarrow X$ be a linear mapping from an associative algebra into an A-bimodule. Then, the following statements are equivalent:

(a) $\delta$ is a derivation and $\delta([a, b])=0$, for all $a, b \in A$;

(b) $\delta$ is an anti-derivation and $\delta([a, b])=0$, for all $a, b \in A$.

Proof The equivalence is clear by just observing that $\delta([a, b])=0$, for all $a, b \in A$ if and only if $\delta(a b)=\delta(b a)$, for all $a, b \in A$.

A central result in the theory of derivations on $\mathrm{C}^{*}$-algebras was established by Ringrose who proved that every (associative) derivation from a $\mathrm{C}^{*}$-algebra $A$ to a Banach $A$-bimodule $X$ is (automatically) continuous (compare [44]). Johnson established in [26] another result to have in mind by proving that every bounded Jordan derivation from a $C^{*}$-algebra $A$ into a Banach $A$-bimodule $X$ is an associative derivation. By a result due to Russo and the third author of this note, we know that every Jordan derivation from $A$ into $X$ is continuous [42, Corollary 17]; consequently, every Jordan derivation from $A$ into $X$ is a derivation.

Let $\delta: A \rightarrow X$ be an anti-derivation from a $C^{*}$-algebra into a Banach $A$-bimodule. It is clear that $\delta(a \circ b)=\delta(a) \circ b+a \circ \delta(b)(a, b \in A)$, and hence, $\delta$ is a Jordan derivation. It follows from the arguments in the previous paragraph that $\delta$ is a continuous derivation. So, every anti-derivation from a $C^{*}$-algebra $A$ into a Banach $A$-bimodule is continuous and a derivation; therefore, a linear mapping $T: A \rightarrow X$ is an anti-derivation if and only if it is a derivation and $T([a, b])=0$ for all $a, b \in A$. So, the natural question is whether there exist non-trivial derivations vanishing on all commutators.

Let $A$ be a $C^{*}$-algebra. According to the usual notation, we write $\mathfrak{c}(A):=\{[a, b]$ : $a, b \in A\}$. The available literature contains a wide list of papers conducted to determine when an element in a $C^{*}$-algebra can be expressed as a finite sum of commutators (see, 
for example, $[14,15,19,36-38,43]$ and references therein). Let us remark two concrete results Fack and de la Harpe showed in [15] that in any finite von Neumann algebra $M$ with central trace $\tau$, an element $x \in M$ with $\tau(x)=0$ can be expressed as a sum of ten commutators. In another remarkable result, Halpern proved that every element $a$ in a properly infinite von Neumann algebra $M$ can be written as the sum of two commutators (cf. [19, Theorem 3.10]).

Let $X$ be a Banach $A$-bimodule, where $A$ is a $C^{*}$-algebra. In this note, we shall deal with the bidual, $X^{* *}$, of $X$, and we shall regard it as a Banach $A^{* *}$-bimodule. For this purpose, we shall refresh our knowledge on Arens extensions and Arens regularity (cf. [3]). Let $m: X \times Y \rightarrow Z$ be a bounded bilinear map where $X$, $Y$ and $Z$ are Banach spaces. According to the construction defined by R. Arens, we define $m^{*}\left(z^{\prime}, x\right)(y):=z^{\prime}(m(x, y))\left(x \in X, y \in Y, z^{\prime} \in Z^{*}\right)$. We obtain in this way a bounded bilinear mapping $m^{*}: Z^{*} \times X \rightarrow Y^{*}$. The same method can be applied to define $m^{* *}=\left(m^{*}\right)^{*}$ and $m^{* * *}: X^{* *} \times Y^{* *} \rightarrow Z^{* *}$. The mapping $x^{\prime \prime} \mapsto m^{* * *}\left(x^{\prime \prime}, y^{\prime \prime}\right)$ is weak* to weak ${ }^{*}$ continuous whenever we fix $y^{\prime \prime} \in Y^{* *}$, and the mapping $y^{\prime \prime} \mapsto m^{* * *}\left(x, y^{\prime \prime}\right)$ is weak* to weak* continuous for every $x \in X$. The previous construction can be applied to the transposed mapping $m^{t}: Y \times X \rightarrow Z$, $m^{t}(y, x)=m(x, y)$, and we define an extension $m^{t * * * t}: X^{* *} \times Y^{* *} \rightarrow Z^{* *}$. Now, the mapping $x^{\prime \prime} \mapsto m^{t * * * t}\left(x^{\prime \prime}, y\right)$ is weak* to weak* continuous whenever we fix $y \in Y$, and the mapping $y^{\prime \prime} \mapsto m^{t * * * t}\left(x^{\prime \prime}, y^{\prime \prime}\right)$ is weak ${ }^{*}$ to weak ${ }^{*}$ continuous for every $x^{\prime \prime} \in X^{* *}$. It should be remarked that the mappings $m^{t * * * t}$ and $m^{* * *}$ need not coincide in general (cf. [3]). The mapping $m$ is called Arens regular if $m^{t * * * t}=m^{* * *}$. One of the best known examples of Arens regular maps is given by the product of any $C^{*}$-algebra. That is, every $C^{*}$-algebra $A$ is Arens regular and the unique Arens extension of the product of $A$ to $A^{* *} \times A^{* *}$ coincides with the product of its enveloping von Neumann algebra (cf. [11, Corollary 3.2.37]).

It is worth to recall some notions. Two projections $p$ and $q$ in a von Neumann algebra $M$ are called (Murray-von Neumann) equivalent if there is a partial isometry $e \in M$ such that $e^{*} e=p$ and $e e^{*}=q$. We write this fact as $p \sim q$. A projection $p$ in $M$ is said to be finite if there is no projection $q<p$ that is equivalent to $p$. A projection $p$ in $M$ is infinite if it is not finite, and properly infinite if $p \neq 0$ and $z p$ is infinite whenever $z$ is a central projection such that $z p \neq 0$ (cf. [46, Definition V.1.15]). The von Neumann algebra $M$ is said to be finite, infinite or properly infinite according to the property of its identity [46, Definition V.1.16].

Let $M$ be a von Neumann algebra. A (faithful) center-valued trace on $M$ is a linear mapping $\tau$ from $M$ onto its center $Z(M)$ satisfying:

(a) $\tau\left(x^{*} x\right)=\tau\left(x x^{*}\right) \geq 0$ for all $x \in M$;

(b) $\tau(a x)=a \tau(x)$, for all $a \in Z(M), x \in M$;

(c) $\tau(1)=1$;

(d) $\tau\left(x^{*} x\right) \neq 0$ for every nonzero $x \in M$.

A von Neumann algebra $M$ is finite if and only if it admits a faithful center-valued trace (which is further weak* continuous) [46, Theorem V.2.6].

Suppose $X$ is a Banach $A$-bimodule over a $C^{*}$-algebra $A$. Let $\pi_{1}: A \times X \rightarrow X$ and $\pi_{2}: X \times A \rightarrow X$ stand for the corresponding module operations given by $\pi_{1}(a, x)=a x$ and $\pi_{2}(x, a)=x a$, respectively. Given $a \in A^{* *}$ and $z \in X^{* *}$, we 
shall write $a z=\pi_{1}^{* * *}(a, z)$ and $z a=\pi_{2}^{* * *}(z, a)$. It is known that $X^{* *}$ is a Banach $A^{* *}$-bimodule (and also a Banach $A$-bimodule) for the just defined operations ([11, Theorem 2.6.15(iii)]). An additional property of this construction tells that

$$
\begin{aligned}
& a x=\pi_{1}^{* * *}(a, x)=w^{*}-\lim _{\lambda} w^{*}-\lim _{\mu} a_{\lambda} x_{\mu}, \text { and } \\
& x a=\pi_{2}^{* * *}(x, a)=w^{*}-\lim _{\mu} w^{*}-\lim _{\lambda} x_{\mu} a_{\lambda},
\end{aligned}
$$

in the weak* topology of $X^{* *}$, whenever $\left(a_{\lambda}\right)$ and $\left(x_{\mu}\right)$ are nets in $A$ and $X$, respectively, such that $a_{\lambda} \rightarrow a \in A^{* *}$ in the weak* topology of $A^{* *}$ and $x_{\mu} \rightarrow x \in X^{* *}$ in the weak* topology of $X^{* *}$ (cf. [11, (2.6.26)]). The reader should be warned that the module operations on $X^{* *}$ need not be separately weak* continuous. This handicap produces some difficulties in our arguments.

Let $d: A \rightarrow M$ be an (anti-)derivation from a $C^{*}$-algebra into a Banach $A$ bimodule. We have already seen that $d$ is continuous and hence $d^{* *}: A^{* *} \rightarrow X^{* *}$ is weak* continuous. It follows from (2.3) that $d^{* *}$ is (anti-)derivation.

It is clear from the above that in a $C^{*}$-algebra where every element coincides with a finite sum of commutators, every anti-derivation is zero. In particular, every antiderivation from a properly infinite von Neumann algebra $M$ into a Banach $M$-bimodule is zero by Halpern's theorem [19, Theorem 3.10]. We can improve this conclusion in the next result.

Theorem 4 Let $\delta: A \rightarrow X$ be an anti-derivation from a $C^{*}$-algebra to a Banach $A$-bimodule. Then, there exists a finite central projection $p_{1}$ in $A^{* *}$ and an element $x_{0} \in X^{* *}$ such that

$$
\delta^{* *}(x)=\delta_{x_{0}}\left(\tau\left(p_{1} x\right)\right)=\left[\tau\left(p_{1} x\right), x_{0}\right], \text { for all } x \in A^{* *},
$$

where $\tau: p_{1} A^{* *} \rightarrow Z\left(p_{1} A^{* *}\right)$ is the (faithful) center-valued trace on $p_{1} A^{* *}$.

Furthermore, if we also assume that $z x=x z$ for all $z \in Z\left(A^{* *}\right)$ and $x \in X^{* *}$ (for example, when $X=A$ or $A^{*}$ or $\left.A^{* *}\right)$, every anti-derivation $\delta: A \rightarrow X$ is zero.

Proof We have already commented in previous paragraphs that $\delta$ is a continuous derivation with $\delta([a, b])=0$ for all $a, b \in A$. Furthermore, $\delta^{* *}: A^{* *} \rightarrow X^{* *}$ is an anti-derivation too. Therefore $\delta^{* *}$ is a derivation and vanishes on every commutator of $A^{* *}$.

By [46, Theorem V.1.19 and Lemma V.1.7], the identity of the von Neumann algebra $A^{* *}$ is uniquely written as the sum of (centrally) orthogonal projections $p_{1}$ and $p_{2}$ such that $p_{1}$ is finite and $p_{2}$ is properly infinite. It follows that $A^{* *}$ decomposes as the orthogonal direct sum of $M_{1}=p_{1} A^{* *} p_{1}$ and $M_{2}=p_{2} A^{* *} p_{2}, M_{1}$ is finite and $M_{2}$ is properly infinite. By Theorem 3.10 in [19], every element $a_{2}$ in $M_{2}$ can be written as the sum of two commutators in $M_{2}$. Furthermore, let $\tau: M_{1} \rightarrow Z\left(M_{1}\right)$ be the faithful center-valued trace of the finite von Neumann algebra $M_{1}$. Theorem 3.2 in [15] asserts that every element $b_{1}$ in $M_{1}$ with $\tau\left(b_{1}\right)=0$ coincides with the sum of ten commutators in $M_{1}$. Since for each $a_{1} \in M_{1}$, the element $b_{1}=a_{1}-\tau\left(a_{1}\right) \in M_{1}$ has zero trace, we deduce that $a_{1}-\tau\left(a_{1}\right)$ writes as the sum of ten commutators in $M_{1}$. 
Since commutators in $A^{* *}$ are sums of commutators in $M_{1}$ and $M_{2}$, we deduce from the properties of $\delta^{* *}$ that

$$
\delta^{* *}\left(a_{1}+a_{2}\right)=\delta^{* *}\left(\tau\left(a_{1}\right)\right), \text { for all } a_{1} \in M_{1}, a_{2} \in M_{2}
$$

On the other hand, $X^{* *}$ is a dual $Z\left(M_{1}\right)$-bimodule with respect to the restricted bimodule operations, and $\left.\delta^{* *}\right|_{Z\left(M_{1}\right)}: Z\left(M_{1}\right) \rightarrow X^{* *}$ is a derivation and an antiderivation. Since every commutative $C^{*}$-algebra $\mathcal{C}$ is amenable (i.e., for every Banach $\mathcal{C}$-bimodule $Y$, every derivation from $\mathcal{C}$ into $Y^{*}$ is inner (see, for example, [11, Theorem 5.6.2(i)]), there exists $x_{0} \in X^{* *}$ such that $\delta^{* *}(z)=\left[z, x_{0}\right]$ for all $z \in Z\left(M_{1}\right)$. This finishes the proof of the first statement.

Let us deal with the last statement. Suppose that $z x=x z$ for all $z \in Z\left(A^{* *}\right)$ and $x \in X^{* *}$. In this case, $\delta^{* *}(z)=\left[z, x_{0}\right]=0$ for all $z \in Z\left(M_{1}\right)$, and thus, $\delta=0$.

Let $A=C[0,1]$ and $X=\mathbb{C}$ equipped with the bimodule operations defined by $f \cdot \lambda:=f(0) \lambda$ and $\lambda \cdot f:=f(1) \lambda$. According to this structure, there exist elements $f \in C[0,1]$ such that $f \cdot 1 \neq 1 \cdot f$. The mapping $\delta_{1}: C[0,1] \rightarrow X$ is a nonzero anti-derivation.

\section{Linear Maps Anti-Derivable at Zero}

Let $A$ and $B$ be $C^{*}$-algebras. It is known that every bounded bilinear form $V: A \times$ $B \rightarrow \mathbb{C}$ admits a unique norm preserving separately weak* continuous extension to $A^{* *} \times B^{* *}$ (cf. [27, Lemma 2.1]). Actually, the same conclusion also holds when $A$ and $B$ are $\mathrm{JB}^{*}$-triples (see [41, Lemma 1]).

Along this note, the self-adjoint part of a $\mathrm{C}^{*}$-algebra $A$ will be denoted by $A_{s a}$.

Let $A$ be a Banach algebra. If instead of requiring $A$ to be zero product determined we only request that for every Banach space $Y$ and every continuous bilinear form $V: A \times A \rightarrow Y$ satisfying $V(a, b)=0$ for every $a, b \in A_{s a}$ with $a b=0$, there exist continuous functionals $\phi, \varphi \in A^{*}$ such that $V(a, b)=\phi(a b)+\varphi(b a)$ for all $a, b \in A$, a celebrated theorem due to Goldstein (see [17, Theorem 1.10]) affirms that every $C^{*}$-algebra satisfies this latter property. This is one of the advantages in the study of derivations on $\mathrm{C}^{*}$-algebras.

Let $X$ be a Banach $A$-bimodule over a Banach algebra $A$. According to the usual terminology, we shall say that $X$ is essential if the linear span of the set $\{a x b: a, b \in$ $A, x \in X\}$ is dense in $X$.

If $A$ is a non-unital $C^{*}$-algebra, 1 denotes the unit in $A^{* *}$, and $\left(u_{\lambda}\right)$ is a bounded approximate unit in $A$ (cf. [40, Theorem 1.4.2]), it is known that $\left(u_{\lambda}\right) \rightarrow 1$ in the weak* topology of $A^{* *}$. Furthermore, if we regard $X^{* *}$ as a Banach $A^{* *}$-bimodule, it follows from the basic properties commented in the first section that

$$
(\eta a) 1=w^{*}-\lim _{\lambda}(\eta a) u_{\lambda}=w^{*}-\lim _{\lambda} \eta\left(a u_{\lambda}\right)=\|\cdot\|-\lim _{\lambda} \eta\left(a u_{\lambda}\right)=\eta a
$$

for all $a \in A$ and $\eta \in X$. Assuming that $X$ is essential, we get $\eta 1=\pi_{2}^{* * *}(\eta, 1)=\eta$ (and similarly $1 \eta=\pi_{1}^{* * *}(1, \eta)=\eta$ ) for all $\eta \in X$. Actually, $\lim _{\lambda}\left\|\eta u_{\lambda}-\eta\right\|=0=$ 
$\lim _{\lambda}\left\|u_{\lambda} \eta-\eta\right\|$ for all $\eta \in X$. Let us take $\eta \in X^{* *}$, and pick via Goldstine's theorem a bounded net $\left(\eta_{\mu}\right)$ in $X$ converging to $\eta$ in the weak* topology of $X^{* *}$. Since $\pi_{2}^{* * *}(\cdot, 1)$ is weak* continuous, we have

$$
\eta 1=\pi_{2}^{* * *}(\eta, 1)=w^{*}-\lim _{\mu} \pi_{2}^{* * *}\left(\eta_{\mu}, 1\right)=w^{*}-\lim _{\mu} \eta_{\mu} 1=w^{*}-\lim _{\mu} \eta_{\mu}=\eta
$$

Our next result is a modular version of [13, Lemma 2.2].

Lemma 5 Let $X$ be a Banach A-bimodule over a $C^{*}$-algebra A. Let $\xi$ be an element in $X$ satisfying the following property: $h \xi k=0$ for every $h, k \in A_{\text {sa }}$ with $h k=0$. Let 1 denote the unit of $A^{* *}$. Then, the element $\eta=1 \xi 1 \in X^{* *}$ satisfies $a \eta b=a \xi b$, for all $a, b \in A$, and commutes with every element in $A$, that is, $\eta a=a \eta$, for all $a \in A$.

Proof Since $A$ may be non-unital, we shall consider $A^{* *}$ and the space $X^{* *}$ as a Banach $A^{* *}$-bimodule. Let $\eta=1 \xi 1 \in X^{* *}$. By the basic properties of the $A^{* *}$-bimodule $X^{* *}$, we have $a \eta b=a \xi b$ for all $a, b \in A$ and $\eta 1=1 \eta=\eta$.

Let us fix an arbitrary $\phi \in X^{*}$ and define the bounded bilinear form given by $V_{\phi}: A \times A \rightarrow \mathbb{C}, V_{\phi}(a, b)=\phi(a \eta b)=\phi(a \xi b)$. It follows from the hypothesis that $V_{\phi}(h, k)=0$, for every $h, k \in A_{s a}$ with $h k=0$, witnessing that $V_{\phi}$ is an orthogonal form in the sense of Goldstein [17]. Theorem 1.9 in [17] implies the existence of two functionals $\varphi_{1}, \varphi_{2} \in A^{*}$ satisfying $V_{\phi}(a, b)=\varphi_{1}(a b)+\varphi_{2}(b a)$ for all $a, b \in A$. We denote by the same symbol $V_{\phi}$ the (unique) separate weak* continuous extension of $V_{\phi}$ to $A^{* *} \times A^{* *}$. We can therefore conclude that

$$
\phi(a \eta)=V_{\phi}(a, 1)=\varphi_{1}(a)+\varphi_{2}(a)=V_{\phi}(1, a)=\phi(\eta a),
$$

for all $a \in A$. The arbitrariness of $\phi \in X^{*}$ combined with the Hahn-Banach theorem implies that $\eta a=a \eta$, for all $a \in A$.

We can now present our characterization of those continuous linear maps on a $\mathrm{C}^{*}$-algebra which are anti-derivable at zero.

Theorem 6 Let $T: A \rightarrow X$ be a bounded linear operator where $A$ is a $C^{*}$-algebra and $X$ is an essential Banach A-bimodule. Then, the following are equivalent:

(a) $T$ is anti-derivable at zero;

(b) There exists an anti-derivation $d: A \rightarrow X^{* *}$ and an element $\xi \in X^{* *}$ satisfying $\xi a=a \xi, \xi[a, b]=0, T(a b)=b T(a)+T(b) a-b \xi a$, and $T(a)=d(a)+\xi a$, for all $a, b \in A$;

Proof $(a) \Rightarrow(b)$ Suppose $T$ is anti-derivable at zero. Let us pick $h_{1}, k, h_{2} \in A_{s a}$ with $h_{j} k=0$ (and thus $k h_{j}=0$ ) for $j=1,2$, it follows from the hypothesis that $T(k) h_{2}+k T\left(h_{2}\right)=0$ and therefore

$$
h_{1} T(k) h_{2}=h_{1}\left(T(k) h_{2}+k T\left(h_{2}\right)\right)=0 .
$$

This shows that the mapping $T: A \rightarrow X$ satisfies the hypotheses of [4, Theorem 2.11]; we therefore conclude from the just quoted result that $T: A \rightarrow X$ is a generalized 
derivation, that is, there exists $\xi \in X^{* *}$ such that

$$
T(a b)=T(a) b+a T(b)-a \xi b, \quad \forall a, b \in A .
$$

By replacing $\xi$ with $1 \xi 1$, we can assume that $1 \xi=\xi 1=\xi$ and (3.3) holds.

It is not hard to check from (3.3) that the mapping $d: A \rightarrow X^{* *}, d(a)=T(a)-\xi a$ is a derivation satisfying $T(a)=d(a)+\xi a$ for all $a \in A$.

If we pick $h, k \in A_{s a}$ with $h k=0$ (and thus $k h=0$ ). We deduce from the hypothesis that $T(h) k+h T(k)=0$, and by (3.3):

$$
0=T(h k)=T(h) k+h T(k)-h \xi k,
$$

identities which combined give $h \xi k=0$ (for any $h, k \in A_{s a}$ with $h k=0$ ). Lemma 5 guarantees that $\xi a=a \xi$ for all $a \in A$.

We shall next show that $d$ is an anti-derivation. Let $(Y, \odot)$ denote the opposite Banach $A$-bimodule $X^{o p}$, that is, $y \odot a=a y$ and $a \odot y=y a$ for all $a \in A$, $y \in Y$. Let us pick $h_{1}, k, h_{2} \in A_{s a}$ with $h_{j} k=0$ for $j=1,2$. We have seen in (3.3) that $h_{1} \odot T(k) \odot h_{2}=h_{2} T(k) h_{1}=0$. Then, the mapping $\tilde{T}: A \rightarrow Y$, $\tilde{T}(a)=T(a)(a \in A)$ satisfies that $h_{1} \odot \tilde{T}(k) \odot h_{2}=0$ for every $h_{1}, k, h_{2} \in A_{s a}$ with $h_{j} k=0$. We deduce from [4, Theorem 2.11] the existence of $\eta \in X^{* *}$ such that $T(a b)=T(a) \odot b+a \odot T(b)-a \odot \eta \odot b, \quad \forall a, b \in A$, equivalently,

$$
T(a b)=b T(a)+T(b) a-b \eta a, \quad \forall a, b \in A .
$$

Replacing $\eta$ with $1 \eta 1$, we can always assume that $\eta=\eta 1=1 \eta$.

By mimicking the arguments above, fix $h, k \in A_{s a}$ with $h k=0$. We deduce from the hypothesis (with $k h=0$ ) that $T(h) k+h T(k)=0$, and by (3.4)

$$
0=T(k h)=T(h) k+h T(k)-h \eta k .
$$

By combining the previous two identities, we get $h \eta k=0$ for all $h, k \in A_{s a}$ with $h k=0$. A new application of Lemma 5 guarantees that $\eta a=a \eta$ for all $a \in A$.

Now, combining the fact that $\xi$ and $\eta$ commute with any element in $A$, and (3.3) and (3.4) with $a=b$, we have $\eta a^{2}=\xi a^{2}$ for all $a \in A$. Since $A$ is a $C^{*}$-algebra, it follows that $a \eta=\eta a=\xi a=a \xi$ for all $a \in A$. Therefore, there is no loss of generality in assuming $\xi=\eta$ in (3.3) and (3.4).

Now, let us apply (3.4) and (3.3) to deduce the following identities:

$$
T(a b)=T(b) a+b T(a)-b \xi a, \text { and } T(b a)=T(b) a+b T(a)-b \xi a,
$$

for all $a, b \in A$. Therefore,

$$
T([a, b])=T(a b-b a)=0 \text { for all } a, b \in A .
$$


Let us analyze the identity (3.3). Let $\left(u_{\lambda}\right)$ be an approximate unit in $A$. Since the identity

$$
T\left(u_{\lambda} b\right)=T\left(u_{\lambda}\right) b+u_{\lambda} T(b)-u_{\lambda} \xi b
$$

holds for every $\lambda, T^{* *}$ is weak* continuous (and hence $T^{* *}\left(u_{\lambda}\right) \rightarrow T^{* *}(1)$ in the weak* topology), $u_{\lambda} T(b) \rightarrow T(b)$ in norm because $X$ is essential, the product of $A^{* *}$ is separately weak ${ }^{*}$ continuous [45, Theorem 1.7.8], $\pi_{1}^{* * *}(\cdot, \xi b)$ is weak ${ }^{*}$ continuous (and thus $u_{\lambda} \xi b=\pi_{1}^{* * *}\left(u_{\lambda}, \xi b\right) \rightarrow \pi_{1}^{* * *}(1, \xi b)=1 \xi b=\xi b$ in the weak ${ }^{*}$ topology), we conclude that

$$
T(b)=T^{* *}(1) b+T(b)-1 \xi b, \text { or equivalently, } T^{* *}(1) b=1 \xi b=\xi b,
$$

for all $b \in A$.

Let us recall that a continuous bilinear mapping $V: A \times A \rightarrow X$ preserves zero products if

$$
a b=0 \text { in } A \Rightarrow V(a, b)=0 .
$$

By [1, Example 1.3(2.), Theorem 2.11 and Definition 2.2], every continuous bilinear mapping $V$ preserving zero products satisfies $V(a b, c)=V(a, b c)$ for all $a, b, c \in A$. By hypothesis, the mapping $V(a, b):=T(b) a+b T(a)$ is continuous and preserves zero products and therefore

$$
T(c) a b+c T(a b)=V(a b, c)=V(a, b c)=T(b c) a+b c T(a),
$$

for all $a, b, c \in A$. If in the above equality we replace $c$ with $u_{\lambda}$, where $\left(u_{\lambda}\right)$ is an approximate unit in $A$, and we take weak* limits, we get

$$
T^{* *}(1) a b+T(a b)=T(b) a+b T(a), \text { for all } a, b \in A \text {. }
$$

Since $\xi a b=T^{* *}(1) a b$ (cf. (3.6)) and $T(a b)=T(b a)=d(b a)+\xi b a$, it follows that

$$
\xi a b+d(b a)+\xi b a=d(b) a+\xi b a+b d(a)+\xi b a=d(b a)+2 \xi b a,
$$

witnessing that $\xi[a, b]=0$.

Therefore, by (3.5)

$$
d([a, b])=T([a, b])-\xi[a, b]=0 \text { for all } a, b \in A .
$$

Lemma 3 proves that $d$ is an anti-derivation.

$(b) \Rightarrow(a)$ Suppose there exist an anti-derivation $d: A \rightarrow X^{* *}$ and an element $\xi \in X^{* *}$ satisfying the stated properties. Let us take $a, b \in A$ with $a b=0$. It follows from the assumptions that 
$T(b) a+b T(a)=(d(b)+\xi b) a+b(d(a)+\xi a)=d(a b)+2 \xi b a=0+2 \xi a b=0$.

Remark 7 If in Theorem 6 the $C^{*}$-algebra $A$ is unital or if $X$ is a dual Banach $A$ bimodule, statement $(b)$ can be replaced with the following:

( $\left.\mathrm{b}^{\prime}\right)$ There exist an anti-derivation $d: A \rightarrow X$ and an element $\xi \in X$ satisfying $\xi a=a \xi, \xi[a, b]=0, T(a b)=b T(a)+T(b) a-b \xi a$, and $T(a)=d(a)+\xi a$, for all $a, b \in A$.

A closer look at the proof of Theorem 6 shows that the desired statement will follow as soon as we prove that the element $\xi$ lies in $X$. If $A$ is unital, this is clear because $T(1)=d(1)+\xi=\xi \in X$. If $X$ is a dual Banach space, we can repeat the arguments in the proof of [1, Proposition 4.3 or Theorem 4.6].

Every $C^{*}$-algebra $A$ is an essential $A$-bimodule because it admits a bounded approximate unit (see [40, Theorem 1.4.2]). The second dual, $A^{* *}$, of $A$ is an $A$-bimodule with respect to the natural product. In general, $A^{* *}$ need not be an essential $A$-bimodule, consider, for example, $A=c_{0}$ and $A^{* *}=\ell_{\infty}$. However, if $A$ is unital, $A^{* *}$ is an essential $A$-bimodule. Despite that $A^{* *}$ is not in general an essential $A$-bimodule, $A$ is weak* dense in $A^{* *}$ by Goldstine's theorem, and it is known that $A$ admits a bounded approximate unit (see [40, Theorem 1.4.2]) which converges to the unit of $A^{* *}$ in the weak* topology. Applying these special properties, the proofs of [4, Lemma 2.10, Theorem 2.11] remain valid to characterize when a bounded linear operator $T: A \rightarrow A^{* *}$ is a generalized derivation. Therefore, the proof of Theorem 6 can be combined with Theorem 4 to get the following result:

Theorem 8 Let $T: A \rightarrow A^{* *}$ be a bounded linear operator where $A$ is a $C^{*}$-algebra. Then, the following are equivalent:

(a) $T$ is anti-derivable at zero;

(b) There exists $\xi \in A^{* *}$ satisfying $\xi a=a \xi, \xi[a, b]=0$, and $T(a)=\xi a$, for all $a, b \in A$.

The preceding theorem can be regarded as a generalization of [16, Corollary 3.8 $(i)]$.

\section{Linear Maps *-Anti-Derivable at Zero}

In this section, we shall deal with continuous linear maps which are $\mathrm{r}^{*}$-anti-derivable at zero. We shall first recall the basic theory on bimodules equipped with an involution. Let $X$ be a Banach $A$-bimodule over a $C^{*}$-algebra $A$. By an $A$-bimodule involution on $X$, we mean a continuous conjugate linear mapping $X \rightarrow X, x \mapsto x^{*}$, satisfying $\left(x^{*}\right)^{*}=x,(a x)^{*}=x^{*} a^{*}$, and $(x a)^{*}=a^{*} x^{*}$, for all $a \in A, x \in X$. The natural involutions on $A$ and on $A^{* *}$ are $A$-bimodule involutions when $A$ and $A^{* *}$ are regarded as Banach $A$-bimodules. Another typical example can be given in the following way: For each functional $\varphi \in A^{*}$ and $a \in A$, the functionals $a \varphi, \varphi a \in A^{*}$ are defined by $(a \varphi)(b)=\varphi(b a)$, and $(\varphi a)(b)=\varphi(a b)$, for all $b \in A$, respectively. These operations 
define a structure of Banach $A$-bimodule on $A^{*}$. Furthermore, for each $\varphi \in A^{*}$ we define $\varphi^{*} \in A^{*}$ by $\varphi^{*}(b):=\overline{\varphi\left(b^{*}\right)}(\forall b \in A)$. It is easy to check that $(a \varphi)^{*}=\varphi^{*} a^{*}$ and $(\varphi a)^{*}=a^{*} \varphi^{*}$ for all $a \in A, \varphi \in A^{*}$. Therefore, $\varphi \mapsto \varphi^{*}$ defines an $A$-bimodule involution on $A^{*}$.

Suppose $x \mapsto x^{*}$ is an $A$-bimodule involution on a Banach $A$-bimodule $X$. We shall regard $X^{*}$ as a Banach $A$-bimodule with module operations given by $(a \phi)(x)=$ $\phi(x a)$, and $(\phi a)(x)=\phi(a x)$, for all $a \in A, x \in X$ and $\phi \in X^{*}$. We shall consider the natural involutions on $X$ and $X^{* *}$ naturally induced by the $A$-bimodule involution of $X$, defined by $\phi^{*}(x):=\overline{\phi\left(x^{*}\right)}\left(\forall \phi \in X^{*}, x \in X\right)$ and $z^{*}(\phi):=\overline{z\left(\phi^{*}\right)}(\forall \phi \in$ $\left.X^{*}, z \in X^{* *}\right)$. Clearly, the involution $z \mapsto z^{*}$ is weak continuous on $X^{* *}$. Let $a \in A$, $z \in X^{* *}$, and let $\left(x_{\mu}\right) \subset X$ a bounded net converging to $z$ in the weak* topology of $X^{* *}$. By the properties of the module operation on $X^{* *}$ (see page 7 ), we have

$$
\begin{aligned}
(a z)^{*} & =\pi_{1}^{* * *}(a, z)^{*}=w^{*}-\lim _{\mu} \pi_{1}\left(a, x_{\mu}\right)^{*}=w^{*}-\lim _{\mu} \pi_{2}\left(x_{\mu}^{*}, a^{*}\right) \\
& =\pi_{2}^{* * *}\left(z^{*}, a^{*}\right)=z^{*} a^{*}
\end{aligned}
$$

and

$$
\begin{aligned}
(z a)^{*} & =\pi_{2}^{* * *}(z, a)^{*}=w^{*}-\lim _{\mu} \pi_{2}\left(x_{\mu}, a\right)^{*}=w^{*}-\lim _{\mu} \pi_{1}\left(a^{*}, x_{\mu}^{*}\right) \\
& =\pi_{1}^{* * *}\left(a^{*}, z^{*}\right)=a^{*} z^{*}
\end{aligned}
$$

A Banach $A$-bimodule equipped with an $A$-bimodule involution will be called a Banach ${ }^{*}$-A-bimodule. Along this section, $X$ will stand for a Banach ${ }^{*}$-A-bimodule over a $C^{*}$-algebra $A$. A linear mapping $T: A \rightarrow X$ will be called $r$ - $^{*}$-anti-derivable at zero (respectively, $l$ - $^{*}$-anti-derivable at zero) if $a b^{*}=0$ in $A$ implies $T(b)^{*} a+$ $b^{*} T(a)=0$ in $X$ (respectively, $a^{*} b=0$ in $A \Rightarrow T(b) a^{*}+b T(a)^{*}=0$ in $X$ ). It is easy to see that $T$ is $\mathrm{r}^{*}$-anti-derivable at zero if and only if the mapping $S: A \rightarrow X$, $S(a):=T\left(a^{*}\right)^{*}(\forall a \in A)$ is 1 - $^{*}$-anti-derivable at zero.

We can now state our main conclusion for continuous linear maps which are $\mathrm{r}^{*}$ anti-derivable at zero.

Theorem 9 Let $T: A \rightarrow X$ be a bounded linear operator where A is a $C^{*}$-algebra and $X$ is an essential Banach ${ }^{*}$-A-bimodule. Then, the following statements are equivalent:

(a) $T$ is $r_{-}^{-}$-anti-derivable at zero (i.e., $a b^{*}=0$ in $A \Rightarrow T(b)^{*} a+b^{*} T(a)=0$ in $X)$;

(b) There exists $a^{*}$-derivation $d: A \rightarrow X^{* *}$ and an element $\xi \in X^{* *}$ satisfying the following properties:

(i) $d([a, b])+[a, b] \xi+\xi^{*}[a, b]=0$, for all $a, b \in A$;

(ii) $T(a b)=a T(b)+T(a) b-a \xi b$, and $T(a)=d(a)+a \xi$ for all $a, b \in A$.

If we further assume that $A$ is unital or $X$ is a dual Banach A-bimodule, we can replace (b) with the following:

$\left(b^{\prime}\right)$ There exists $a^{*}$-derivation $d: A \rightarrow X$ and an element $\xi \in X$ satisfying the properties (i)-(ii) above. 


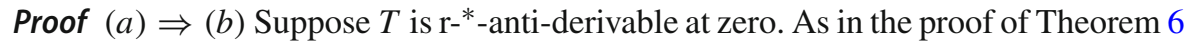
we observe that given $h_{1}, h_{2}, k \in A_{s a}$ with $h_{j} k=0$ for $j=1,2$, we have $T\left(h_{1}\right)^{*} k+$ $h_{1} T(k)=0$, and consequently,

$$
0=\left(T\left(h_{1}\right)^{*} k+h_{1} T(k)\right) h_{2}=h_{1} T(k) h_{2} .
$$

Theorem 2.11 in [4] assures that $T$ is a generalized derivation, that is, there exists an element $\xi \in X^{* *}$ satisfying

$$
T(a b)=T(a) b+a T(b)-a \xi b, \quad \forall a, b \in A .
$$

By replacing $\xi$ with $1 \xi 1$, we can assume that $\xi=1 \xi=\xi 1$. It is routine to check that the mapping $d: A \rightarrow X^{* *}, d(a)=T(a)-a \xi$ is a derivation and $T(a)=d(a)+a \xi$ for all $a \in A$.

The same arguments employed in the proof of Theorem 6 (3.6) prove that

$$
T^{* *}(1) b=1 \xi b=\xi b, \text { for all } b \in A .
$$

We consider the continuous bilinear mapping $V: A \times A \rightarrow X$ defined by $V(a, b):=T\left(b^{*}\right)^{*} a+b T(a)$. If $a\left(b^{*}\right)^{*}=a b=0$, the hypothesis implies that $V(a, b)=0$. Therefore, $V$ preserves zero products. We conclude from [1, Example 1.3(2.), Theorem 2.11 and Definition 2.2] that $V(a b, c)=V(a, b c)$, or equivalently,

$$
T\left(c^{*}\right)^{*} a b+c T(a b)=T\left(c^{*} b^{*}\right)^{*} a+b c T(a)
$$

for all $a, b, c \in A$. So, if $\left(u_{\lambda}\right)$ is an approximate unit in $A$, we have

$$
T\left(u_{\lambda}\right)^{*} a b+u_{\lambda} T(a b)=T\left(u_{\lambda} b^{*}\right)^{*} a+b u_{\lambda} T(a),
$$

for all $\lambda, a, b \in A$. We can take norm limits on the right- hand side. For the lefthand side, we observe that the bimodule operations $\pi_{2}^{* * *}(\cdot, a b)$ and $\pi_{1}^{* * *}(\cdot, T(a b))$ are weak ${ }^{*}$ continuous. Taking weak ${ }^{*}$ limits in $\lambda$ in the previous equality, we derive

$$
T^{* *}(1)^{*} a b+T(a b)=T^{* *}(1)^{*} a b+1 T(a b)=T\left(b^{*}\right)^{*} a+b T(a),
$$

and

$$
b^{*} a^{*} T^{* *}(1)+T(a b)^{*}=a^{*} T\left(b^{*}\right)+T(a)^{*} b^{*},
$$

for all $a, b \in A$. Replacing $a$ with $u_{\lambda}$, we get

$$
b^{*} u_{\lambda} T^{* *}(1)+T\left(u_{\lambda} b\right)^{*}=u_{\lambda} T\left(b^{*}\right)+T\left(u_{\lambda}\right)^{*} b^{*}
$$

for all $\lambda, b \in A$. Now, taking weak* limits in $\lambda$ we obtain

$$
b^{*} T^{* *}(1)+T(b)^{*}=T\left(b^{*}\right)+T^{* *}(1)^{*} b^{*},
$$


equivalently,

$$
T(b)^{*}-T^{* *}(1)^{*} b^{*}=T\left(b^{*}\right)-b^{*} T^{* *}(1) \text {, for all } b \in A \text {. }
$$

Consequently,

$$
T(h)+T^{* *}(1)^{*} h=T(h)^{*}+h T^{* *}(1), \quad \forall h \in A_{s a},
$$

that is,

$$
d(h)+h \xi+T^{* *}(1)^{*} h=d(h)^{*}+\xi^{*} h+h T^{* *}(1), \quad \forall h \in A_{s a} .
$$

Multiplying by $k_{1} \in A_{s a}$ on the left and by $k_{2} \in A_{s a}$ on the right and applying (4.3), we get

$$
\begin{aligned}
k_{1} d(h) k_{2}+k_{1} h \xi k_{2}+k_{1} \xi^{*} h k_{2} & =k_{1} d(h) k_{2}+k_{1} h \xi k_{2}+k_{1} T^{* *}(1)^{*} h k_{2} \\
& =k_{1} d(h)^{*} k_{2}+k_{1} \xi^{*} h k_{2}+k_{1} h T^{* *}(1) k_{2} \\
& =k_{1} d(h)^{*} k_{2}+k_{1} \xi^{*} h k_{2}+k_{1} h \xi k_{2}
\end{aligned}
$$

for all $h, k_{1}, k_{2} \in A_{s a}$. It then follows that

$$
a d(h) b=a d(h)^{*} b, \text { for all } h \in A_{s a}, a, b \in A .
$$

Since the mappings $\pi_{1}^{* * *}(\cdot, d(h) b)$ and $\pi_{1}^{* * *}\left(\cdot, d(h)^{*} b\right)$ are weak* continuous, we can replace $a$ with $u_{\lambda}$ and take weak* limits in $\lambda$ to deduce that

$$
d(h) b=d(h)^{*} b \text {, and } b^{*} d(h)^{*}=b^{*} d(h), \text { for all } h \in A_{s a}, b \in A \text {. }
$$

Now, by the local Gelfand theory, for each $h \in A_{s a}$, there exist $h_{1}, h_{2} \in A_{s a}$ with $h_{1} h_{2}=h_{2} h_{1}=h$. If we apply (4.7) and the fact that $d$ is a derivation, we arrive at

$$
\begin{aligned}
d(h)^{*} & =d\left(h_{1} h_{2}\right)^{*}=\left(d\left(h_{1}\right) h_{2}+h_{1} d\left(h_{2}\right)\right)^{*}=h_{2} d\left(h_{1}\right)^{*}+d\left(h_{2}\right)^{*} h_{1} \\
& =h_{2} d\left(h_{1}\right)+d\left(h_{2}\right) h_{1}=d\left(h_{2} h_{1}\right)=d(h) .
\end{aligned}
$$

The arbitrariness of $h \in A_{s a}$ proves that $d(a)^{*}=d\left(a^{*}\right)$ for all $a \in A$, witnessing that $d$ is a ${ }^{*}$-derivation.

We claim that

$$
d([a, b])+\xi^{*}[a, b]+[a, b] \xi=0 \text { for all } a, b \in A .
$$

Namely, by (4.3) and (4.4) for all $a, b, c \in A$ we have

$$
c \xi^{*} a b+c T(a b)=c T^{* *}(1)^{*} a b+c T(a b)=c T\left(b^{*}\right)^{*} a+c b T(a),
$$


equivalently

$$
\begin{aligned}
c \xi^{*} a b+c d(a b)+c a b \xi & =c d\left(b^{*}\right)^{*} a+c \xi^{*} b a+c b d(a)+c b a \xi \\
& =c d(b a)+c \xi^{*} b a+c b a \xi
\end{aligned}
$$

where in the last equality we applied that $d$ is *-derivation. Therefore,

$$
c d([a, b])+c \xi^{*}[a, b]+c[a, b] \xi=0 \text {, for all } a, b, c \in A .
$$

Replacing $c$ with $u_{\lambda}$, where $\left(u_{\lambda}\right)$ is an approximate unit in $A$, and having in mind that the maps $\pi_{1}^{* * *}(\cdot, d([a, b]))$ and $\pi_{1}^{* * *}\left(\cdot, \xi^{*}[a, b]\right)$ are weak ${ }^{*}$ continuous, by taking weak* limits in $\lambda$ we obtain the identity claimed in (4.8).

$(b) \Rightarrow(a)$ Suppose there exists a *-derivation $d: A \rightarrow X^{* *}$ and an element $\xi \in X^{* *}$ satisfying properties $(i)-(i i)$ in the statement. Let us fix $a, b \in A$ with $a b^{*}=0$. It follows from the assumptions that

$$
\begin{aligned}
T(b)^{*} a+b^{*} T(a) & =d(b)^{*} a+\xi^{*} b^{*} a+b^{*} d(a)+b^{*} a \xi \\
& =d\left(b^{*}\right) a+b^{*} d(a)+\xi^{*} b^{*} a+b^{*} a \xi \\
& =d\left(b^{*} a\right)+\xi^{*} b^{*} a+b^{*} a \xi=d\left(\left[b^{*}, a\right]\right)+\xi^{*}\left[b^{*}, a\right]+\left[b^{*}, a\right] \xi \\
& =(\operatorname{by}(i))=0 .
\end{aligned}
$$

The proof of the last statement can be obtained with the arguments we gave in Remark 7.

As we commented before, for a $C^{*}$-algebra $A$, the proofs of $[4$, Lemma 2.10, Theorem 2.11] remain valid to characterize when a bounded linear operator $T: A \rightarrow$ $A^{* *}$ is a generalized derivation. So, the proofs of Theorem 9 remains valid to get the following result:

Theorem 10 Let $T: A \rightarrow A^{* *}$ be a bounded linear operator, where A is a $C^{*}$-algebra. Then, the following statements are equivalent:

(a) $T$ is $r^{-*}$-anti-derivable at zero (i.e., ab ${ }^{*}=0$ in $A \Rightarrow T(b)^{*} a+b^{*} T(a)=0$ in $\left.A^{* *}\right)$;

(b) There exists $a^{*}$-derivation $d: A \rightarrow A^{* *}$ and an element $\xi \in A^{* *}$ satisfying the following properties:

(i) $d([a, b])+[a, b] \xi+\xi^{*}[a, b]=0$, for all $a, b \in A$;

(ii) $T(a b)=a T(b)+T(a) b-a \xi b$, and $T(a)=d(a)+a \xi$ for all $a, b \in A$.

The description of those continuous linear operators which are 1-*-anti-derivable at zero is a straight consequence of Theorem 9.

Theorem 11 Let $T: A \rightarrow X$ be a bounded linear operator where $A$ is a $C^{*}$-algebra and $X$ is an essential Banach ${ }^{*}-A$-bimodule. Then, the following statements are equivalent: 
(a) $T$ is $l_{-*}$-anti-derivable at zero (i.e., $a^{*} b=0$ in $A \Rightarrow T(b) a^{*}+b T(a)^{*}=0$ in $X)$;

(b) There exists $a^{*}$-derivation $d: A \rightarrow X^{* *}$ and an element $\eta \in X^{* *}$ satisfying the following properties:

(i) $d([a, b])+[a, b] \eta^{*}+\eta[a, b]=0$, for all $a, b \in A$;

(ii) $T(a b)=a T(b)+T(a) b-a \eta b$, and $T(a)=d(a)+\eta a$ for all $a, b \in A$.

If we further assume that $A$ is unital or $X$ is a dual Banach A-bimodule, we can replace (b) with the following:

$\left(b^{\prime}\right)$ There exists $a^{*}$-derivation $d: A \rightarrow X$ and an element $\eta \in X$ satisfying the properties $(i)-(i i)$ above.

Proof By observing that $T$ is $1-^{*}$-anti-derivable at zero if and only if the mapping $S: A \rightarrow X, S(a):=T\left(a^{*}\right)^{*}(\forall a \in A)$ is $\mathrm{r}^{*}$-anti-derivable at zero, an application of Theorem 9 tells that this is the case if and only if there exists a ${ }^{*}$-derivation $d: A \rightarrow$ $X^{* *}$ and an element $\xi \in X^{* *}$ satisfying the following properties:

(i) $d([a, b])+[a, b] \xi+\xi^{*}[a, b]=0$, for all $a, b \in A$;

(ii) $S(a b)=a S(b)+S(a) b-a \xi b$, and $S(a)=d(a)+a \xi$ for all $a, b \in A$.

Taking $\eta=\xi^{*}$, the rest can be straightforwardly checked by the reader.

Corollary 12 Let $T: A \rightarrow A^{* *}$ be a bounded linear operator where $A$ is a $C^{*}$-algebra. Then, the following statements are equivalent:

(a) $T$ is $l$-*-anti-derivable at zero;

(b) There exists $a^{*}$-derivation $d: A \rightarrow A^{* *}$ and an element $\eta \in A^{* *}$ satisfying the following properties:

(i) $d([a, b])+[a, b] \eta^{*}+\eta[a, b]=0$, for all $a, b \in A$;

(ii) $T(a b)=a T(b)+T(a) b-a \eta b$, and $T(a)=d(a)+\eta a$ for all $a, b \in A$.

Acknowledgements A.M. Peralta was partially supported by the Spanish Ministry of Science, Innovation and Universities (MICINN) and European Regional Development Fund Project No. PGC2018-093332-BI00, Junta de Andalucía Grant FQM375 and Proyecto de I+ D + i del Programa Operativo FEDER Andalucia 2014-2020, ref. A-FQM-242-UGR18.

This work was supported by the Deanship of Scientific Research (DSR), King Abdulaziz University. The authors, therefore, gratefully acknowledge DSR technical and financial support.

The results in this paper are part of the first author's PhD thesis at King Abdulaziz University.

We acknowledge the thorough revision made by the anonymous referee including several sharp comments on Theorem 6.

Open Access This article is licensed under a Creative Commons Attribution 4.0 International License, which permits use, sharing, adaptation, distribution and reproduction in any medium or format, as long as you give appropriate credit to the original author(s) and the source, provide a link to the Creative Commons licence, and indicate if changes were made. The images or other third party material in this article are included in the article's Creative Commons licence, unless indicated otherwise in a credit line to the material. If material is not included in the article's Creative Commons licence and your intended use is not permitted by statutory regulation or exceeds the permitted use, you will need to obtain permission directly from the copyright holder. To view a copy of this licence, visit http://creativecommons.org/licenses/by/4.0/. 


\section{References}

1. Alaminos, J., Bresar, M., Extremera, J., Villena, A.: Maps preserving zero products. Stud. Math. 193(2), 131-159 (2009)

2. Araujo, J., Jarosz, K.: Biseparating maps between operator algebras. J. Math. Anal. Appl. 282, 48-55 (2003)

3. Arens, R.: The adjoint of a bilinear operation. Proc. Am. Math. Soc. 2, 839-848 (1951)

4. Ayupov, S., Kudaybergenov, K., Peralta, A.M.: A survey on local and 2-local derivations on C*-and von Neumann algebras, Contemporary Mathematics. Am. Math. Soc. 672, 73-126 (2016)

5. Brešar, M.: Multiplication algebra and maps determined by zero products. Linear Multilinear Algebra 60, 763-768 (2012)

6. Burgos, M., Cabello-Sánchez, J., Peralta, A.M.: Linear maps between $C^{*}$-algebras that are *homomorphisms at a fixed point. Quaest. Math. 42(2), 151-164 (2019)

7. Burgos, M., Fernández-Polo, F.J., Garcés, J., Peralta, A.M.: Local triple derivations on C*-algebras. Commun. Algebra 42(3), 1276-1286 (2014)

8. Burgos, M., Fernández-Polo, F.J., Garcés, J.J., Martínez Moreno, J., Peralta, A.M.: Orthogonality preservers in $\mathrm{C}^{*}$-algebras, JB*-algebras and JB*-triples. J. Math. Anal. Appl. 348, 220-233 (2008)

9. Burgos, M., Fernández-Polo, F.J., Peralta, A.M.: Local triple derivations on $\mathrm{C}^{*}$-algebras and JB*triples. Bull. Lond. Math. Soc. 46(4), 709-724 (2014)

10. Chebotar, M.A., Ke, W.-F., Lee, P.-H., Wong, N.-C.: Mappings preserving zero products. Stud. Math. 155, 77-94 (2003)

11. Dales, H.G.: Banach Algebras and Automatic Continuity. London Mathematical Society Monographs (New Series), vol. 24. Oxford Science Publications, Oxford (2000)

12. Essaleh, A.B.A., Peralta, A.M.: Linear maps on $\mathrm{C}^{*}$-algebras which are derivations or triple derivations at a point. Linear Algebra Appl. 538, 1-21 (2018)

13. Fadaee, B., Ghahramani, H.: Linear maps behaving like derivations or anti-derivations at orthogonal elements on $C^{*}$-algebras. Malays. Math. Sci. Soc, Bull (2019). https://doi.org/10.1007/s40840-01900841-6

14. Fack, Th: Finite sums of commutators in $C^{*}$-algebras. Ann. Inst. Fourier Grenoble 32, 129-137 (1982)

15. Fack, Th, de la Harpe, P.: Sommes de commutateurs dans les alg'ebres de von Neumann finies continues. Ann. Inst. Fourier Grenoble 30, 49-73 (1980)

16. Ghahramani, H., Pan, Z.: Linear maps on *-algebras acting on orthogonal elements like derivations or anti-derivations. Filomat 32(13), 4543-4554 (2018)

17. Goldstein, S.: Stationarity of operator algebras. J. Funct. Anal. 118(2), 275-308 (1993)

18. Goldstein, S., Paszkiewicz, A.: Linear combinations of projections in von Neumann algebras. Proc. Am. Math. Soc. 116, 175-183 (1992)

19. Halpern, H.: Commutators in properly infinite von Neumann algebras. Trans. Am. Math. Soc. 139, 55-73 (1969)

20. Hanche-Olsen, H., Størmer, E.: Jordan Operator Algebras. Pitman, London (1984)

21. Hejazian, S., Niknam, A.: Modules Annihilators and module derivations of JB*-algebras. Indian J. Pure Appl. Math. 27, 129-140 (1996)

22. Hou, J.C., Qi, X.F.: Additive maps derivable at some points on $J$-subspace lattice algebras. Linear Algebra Appl. 429, 1851-1863 (2008)

23. Jamjoom, F.B., Peralta, A.M., Siddiqui, A.A.: Jordan weak amenability and orthogonal forms on JB*-algebras. Banach J. Math. Anal. 9(4), 126-145 (2015)

24. Jing, W.: On Jordan all-derivable points of $B(H)$. Linear Algebra Appl. 430(4), 941-946 (2009)

25. Jing, W., Lu, S.J., Li, P.T.: Characterization of derivations on some operator algebras. Bull. Aust. Math. Soc. 66, 227-232 (2002)

26. Johnson, B.E.: Symmetric amenability and the nonexistence of Lie and Jordan derivations. Math. Proc. Camb. Philos. Soc. 120(3), 455-473 (1996)

27. Johnson, B.E., Kadison, R.V., Ringrose, J.R.: Cohomology of operator algebras III. Reducting to normal cohomology. Bull. Sot. Math. France 100, 73-96 (1972)

28. Leung, C.-W., Tsai, C.-W., Wong, N.-C.: Linear disjointness preservers of $\mathrm{W}^{*}$-algebras. Math. Z. 270, 699-708 (2012)

29. Leung, C.-W., Wong, N.-C.: Zero product preserving linear maps of CCR C*-algebras with Hausdorff spectrum. J. Math. Anal. Appl. 361, 187-194 (2010) 
30. Li, J., Pan, Z.: Annihilator-preserving maps, multipliers, and derivations. Linear Algebra Appl. 423, 5-13 (2010)

31. Lin, Y.-F., Mathieu, M.: Jordan isomorphism of purely infinite C*-algebras. Quart. J. Math. 58, 249-253 (2007)

32. Liu, J.-H., Chou, C.-Y., Liao, C.-J., Wong, N.-C.: Disjointness preservers of AW*-algebras. Linear Algebra Appl. 552, 71-84 (2018)

33. Liu, J.-H., Chou, C.-Y., Liao, C.-J., Wong, N.-C.: Linear disjointness preservers of operator algebras and related structures. Acta Sci. Math. (Szeged) 84(1-2), 277-307 (2018)

34. Lu, F.: Characterizations of derivations and Jordan derivations on Banach algebras. Linear Algebra Appl. 430(8-9), 2233-2239 (2009)

35. Marcoux, L.W.: On the Linear Span of the Projections in Certain Simple $C^{*}$-algebras. Indiana Univ. Math. J. 51, 753-771 (2002)

36. Marcoux, L.W.: Sums of small numbers of commutators. J. Oper. Theory 56, 111-142 (2006)

37. Marcoux, L.W.: Projections, commutators and Lie ideals in $\mathrm{C}^{*}$-algebras. Math. Proc. R. Ir. Acad. 110A, 31-55 (2010)

38. Marcoux, L.W., Murphy, G.J.: Unitarily-invariant linear spaces in C*-algebras. Proc. Am. Math. Soc. 126(12), 3597-3605 (1998)

39. Pearcy, C., Topping, D.: Sum of small numbers of idempotent. Michigan Math. J. 14, 453-465 (1967)

40. Pedersen, G.K.: C*-algebras and their automorphism groups. Academic Press, London (1979)

41. Peralta, A.M., Rodríguez Palacios, A.: Grothendieck's inequalities for real and complex JBW*-triples. Proc. Lond. Math. Soc. (3) 83(3), 605-625 (2001)

42. Peralta, A.M., Russo, B.: Automatic continuity of triple derivations on $\mathrm{C}^{*}$-algebras and JB*-triples. J. Algebra 399, 960-977 (2014)

43. Pop, C.: Finite sums of commutators. Proc. Am. Math. Soc. 130, 3039-3041 (2002)

44. Ringrose, J.R.: Automatic continuity of derivations of operator algebras. J. Lond. Math. Soc. 2(5), 432-438 (1972)

45. Sakai, S.: C*- and W*-Algebras. Springer, Berlin (1971)

46. Takesaki, M.: Theory of Operator Algebras I. Springer, New York (2003)

47. Tsai, C.-W.: The orthogonality structure determines a $C^{*}$-algebra with continuous trace. Oper. Matrices 5, 529-540 (2011)

48. Tsai, C.-W., Wong, N.-C.: Linear orthogonality preservers of standard operator algebras. Taiwan. J. Math. 14, 1047-1053 (2010)

49. Wolff, M.: Disjointness preserving operators in $C^{*}$-algebras. Arch. Math. 62, 248-253 (1994)

50. Wong, N.-C.: Triple homomorphisms of $C^{*}$-algebras. Southeast Asian Bull. Math. 29, 401-407 (2005)

51. Wong, N.-C.: Zero product preservers of $C^{*}$-algebras. Contemp. Math. 435, 377-380 (2007)

52. Zhang, Y.F., Hou, J.C., Qi, X.F.: Characterizing derivations for any nest algebras on Banach space by their behaviors at an injective operator. Linear Algebra Appl. 449(15), 312-333 (2014)

53. Zhang, Y.F., Hou, J.C., Qi, X.F.: All-derivable subsets for nest algebras on Banach spaces. Int. Math. Forum 9(1-4), 1-11 (2014)

54. Zhu, J.: All-derivable points of operator algebras. Linear Algebra Appl. 427, 1-5 (2007)

55. Zhu, J., Xiong, C.P.: Generalized derivable mappings at zero point on nest algebras. Acta Math. Sin. 45, 783-788 (2002)

56. Zhu, J., Xiong, C.P.: Generalized derivable mappings at zero point on some reflexive operator algebras. Linear Algebra Appl. 397, 367-379 (2005)

57. Zhu, J., Xiong, C.P.: Derivable mappings at unit operator on nest algebras. Linear Algebra Appl. 422, 721-735 (2007)

58. Zhu, J., Xiong, C.P.: All-derivable points in continuous nest algebras. J. Math. Anal. Appl. 340, 845853 (2008)

59. Zhu, J., Xiong, Ch., Li, P.: Characterizations of all-derivable points in $B(H)$. Linear Multilinear Algebra 64(8), 1461-1473 (2016)

60. Zhu, J., Zhao, S.: Characterizations all-derivable points in nest algebras. Proc. Am. Math. Soc. 141(7), 2343-2350 (2013)

Publisher's Note Springer Nature remains neutral with regard to jurisdictional claims in published maps and institutional affiliations. 